\title{
Erste Tagung der Akademie der AOTrauma Deutschland
}

\author{
Hans-Jörg Oestern, Karl-Heinz Frosch
}

Die 1. Tagung der Akademie der AOTrauma Deutschland (AOTD) fand am 11. und 12.05.2017 in Münster statt.

An der 1. Tagung nur für Mitglieder der Akademie nahmen 78 Mitglieder teil. In den 2 Sitzungen „Cheese \& Wein Session: Chefarzt offener Erfahrungsaustausch: Was war gut, was schlecht? Was würde ich anders machen?" und „Falldiskussion: Ich habe es doch kommen sehen..." wurden Erfahrungen selbstkritisch vorgetragen und konstruktiv diskutiert. Insbesondere die offene und ehrliche Diskussion wurde sehr geschätzt. In der letzten Sitzung ging es um einen Ausblick in die nähere Zukunft von „Hightech in der Medizin“, insbesondere „Touch Surgery: Das Assistenzarzt-Curriculum in der Hosentasche“ und „Multipurpose Surgical Simulation“.

Abends wurden in gemütlicher Runde die Gespräche vertieft.
Es wurden 16 neue Mitglieder in die Akademie aufgenommen: Thomas Dienstknecht (Düren), Marcel Dudda (Essen), Oliver Gonschorek (Murnau), Georg Gradl (Harlaching), Lars Großterlinden (Hamburg), Benjamin König (Freudenstadt), Markus Kröber (Hamburg), Sven Lendemans (Essen), Martin Lucke (München), Robert Morrison (Ingolstadt), Sabine Ochman (Münster), Oliver Pieske (Oldenburg), Christoph Riepl (Winnenden), Marco Sträter (Datteln), Guido Wanner (Donaueschingen) und Britt Wildemann (Berlin). Insgesamt hat die Akademie 366 Mitglieder.

Die nächste Tagung nur für Mitglieder der Akademie findet am 10. und 11.05.2018 in Erfurt statt.

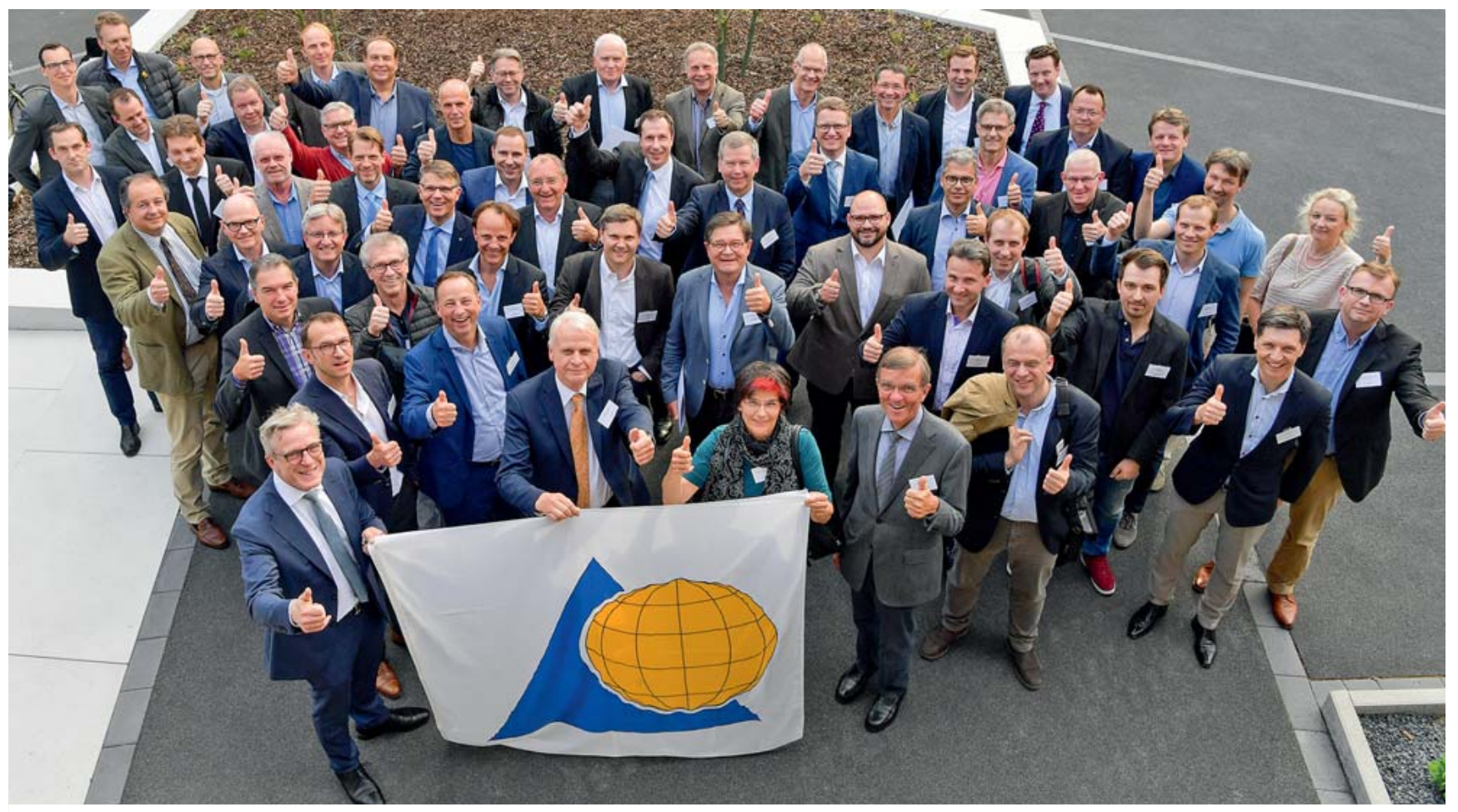

- Abb. 1 Gruppenfoto der Akademietagung. Bild: AO Foundation. 


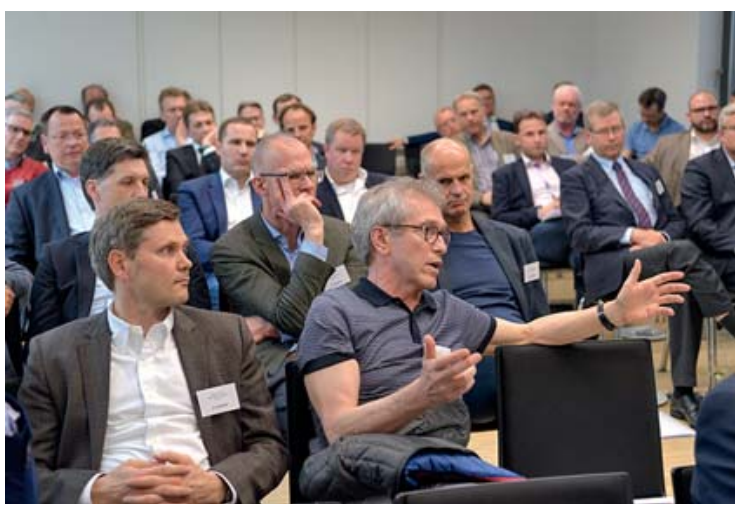

- Abb. 2 Offener Erfahrungsaustausch mit Felix Bonnaire. Bild: AO Foundation.

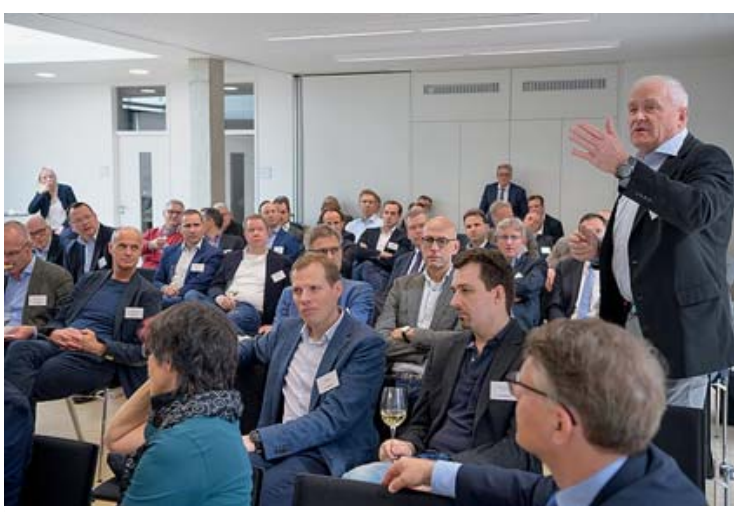

- Abb. 3 Diskussionsbeitrag von Norbert Haas. Bild: AO Foundation.

\section{AOTD-Akademie auf \\ TK Experten-Symposium 2017}

Eine begrenzte Anzahl von Mitgliedern der Akademie der AOTrauma Deutschland (AOTD) wird wie im Vorjahr nach einer Ausschreibung unter den Akademiemitgliedern zur Teilnahme am 12. Europäischen AOTK Experten-Symposium nach Mainz am 03. und 04.11.2017 eingeladen. Die Teilnahme ist nur für Mitglieder der AOTD-Akademie möglich. Die Ausschreibung erfolgt im Spätsommer/ Herbst.

Prof. Dr. Hans-Jörg Oestern, Celle Karl-Heinz Frosch, Hamburg

\section{Bibliografie}

DOI https://doi.org/10.1055/s-0043-116397

OP-JOURNAL 2017; 33: 195-196 @ Georg Thieme Verlag KG Stuttgart · New York ISSN 0178-1715

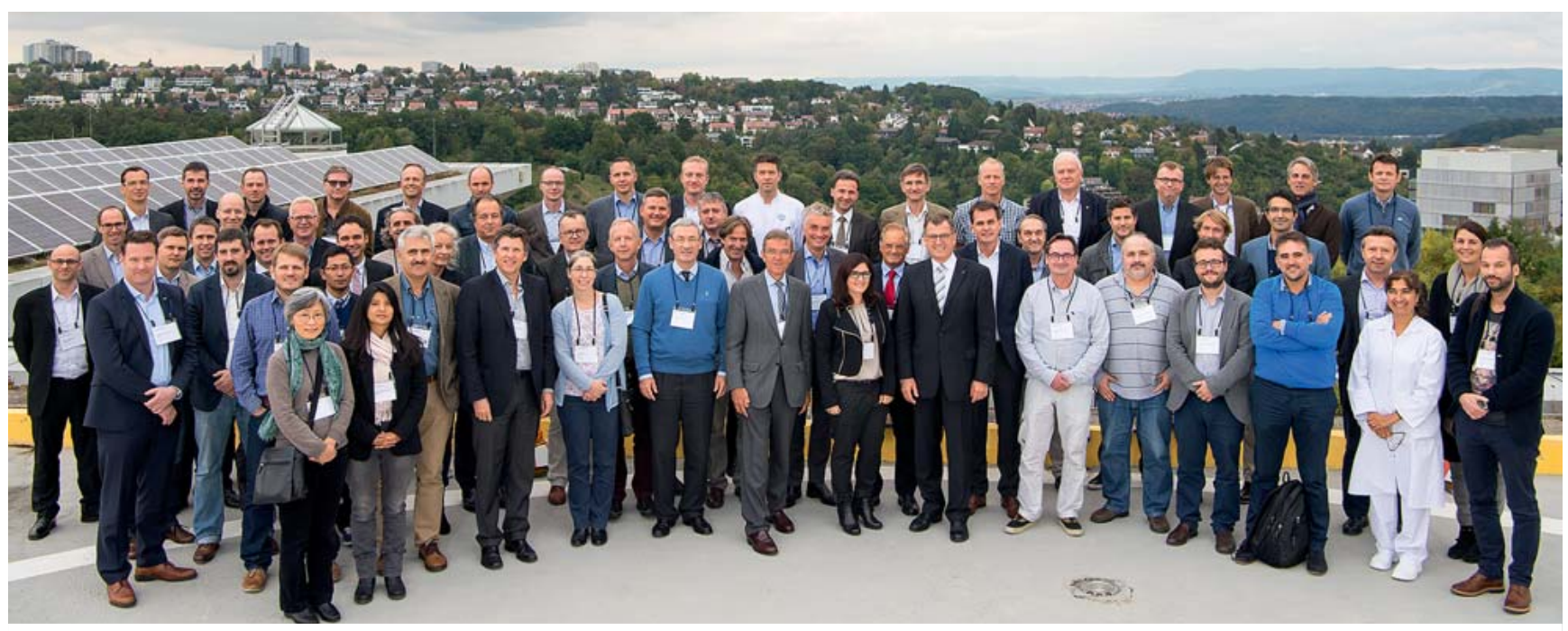

- Abb. 5 Die Teilnehmer des 11. AOTK Experts' Symposium in Tübingen 2016. Bild: Jürgen Staiger, AO Foundation. 